02

\title{
Определение стехиометрии, концентрации ОН-групп и точечных дефектов в кристаллах ниобата лития по ИК спектрам поглощения
}

\author{
(C) Н.А. Теплякова, Н.В. Сидоров, М.Н. Палатников \\ Институт химии и технологии редких элементов и минерального сырья им. И.В. Тананаева \\ Федерального исследовательского центра „Кольский научный центр РАН“, \\ 184209 Апатиты, Мурманская обл., Россия \\ e-mail: n.tepliakova@ksc.ru
}

Поступила в редакцию 20.03.2020 г.

В окончательной редакции 21.04.2020 г.

Принята к публикации 28.04.2020 г.

По ИК спектрам поглощения в области валентных колебаний ОН-групп рассчитаны концентрация

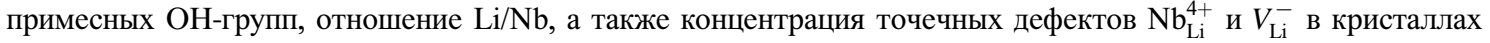
$\mathrm{LiNbO}_{3}$ стехиометрического и конгруэнтного составов, а также в кристаллах, легированных магнием и цинком в концентрациях, близких к пороговым. Обнаружена корреляция в поведении полос в ИК спектрах поглощения сильно легированных кристаллов $\mathrm{LiNbO}_{3}: \mathrm{Mg}$ и $\mathrm{LiNbO}_{3}: \mathrm{Zn}$ с поведением линии спектра комбинационного рассеяния, отвечающей валентным мостиковым колебаниям атомов кислорода в октаэдре $\mathrm{NbO}_{6}$ вдоль полярной оси.

Ключевые слова: кристалл ниобата лития, легирование, ИК спектроскопия поглощения, комбинационное рассеяние света.

DOI: $10.21883 /$ OS.2020.08.49708.85-20

\section{Введение}

Нелинейно-оптические, электрические и фоторефрактивные свойства монокристалла ниобата лития $\left(\mathrm{LiNbO}_{3}\right)$ во многом определяются присутствием в структуре точечных, а также комплексных дефектов, обусловленных наличием водородных связей. При этом состояние дефектности кристалла существенно зависит от стехиометрии (отношения $\mathrm{Li} / \mathrm{Nb}$ ) и наличия легирующих добавок. Многие физические свойства кристалла $\mathrm{LiNbO}_{3}$ (величина коэрцитивного поля, положение фундаментального края оптического поглощения, стойкость к повреждению оптическим излучением и др.) могут быть улучшены путем изменения состояния дефектности его структуры. Кроме того, считается, что некоторые свойства сильнолегированных магнием кристаллов ниобата лития, когда концентрация легирующего элемента близка к пороговому значению, смещаются к свойствам кристаллов стехиометрического состава $(\mathrm{Li} / \mathrm{Nb}=1)$, т. е. тоже определяются отношением $\mathrm{Li} / \mathrm{Nb}[1,2]$. Этот факт является важным, поскольку стехиометрические кристаллы обладают более низким значением коэрцитивного поля по сравнению с конгруэнтными кристаллами $(\mathrm{Li} / \mathrm{Nb}=0.946)$, что делает их перспективными для создания материалов для преобразования лазерного излучения на периодически поляризованных доменах микронных и субмикронных размеров [3].

В кристаллической решетке конгруэнтного кристалла $\mathrm{LiNbO}_{3}$, согласно модели компенсации Li-вакансий [2], присутствует $\sim 1 \mathrm{~mol} \%$ точечных дефектов $\mathrm{Nb}_{\mathrm{Li}}^{4+}$ (избыточные катионы $\mathrm{Nb}^{5+}$, расположенные в позициях катионов $\mathrm{Li}^{+}$идеальной структуры стехиометрического состава $(\mathrm{Li} / \mathrm{Nb}=1))$, и $\sim 4 \mathrm{~mol} \%$ точечных дефектов $V_{\mathrm{Li}}$. В структуре идеального стехиометрического кристалла точечных дефектов $\mathrm{Nb}_{\mathrm{Li}}^{4+}$ и соответственно дефектов $V_{\mathrm{Li}}^{-}$ нет. Наличие точечных дефектов $\mathrm{Nb}_{\mathrm{Li}}$, являющихся глубокими электронными ловушками, существенно влияет на фоторефрактивные свойства как номинально чистых, так и легированных кристаллов $\mathrm{LiNbO}_{3}$ [2,4]. Кроме дефектов $\mathrm{Nb}_{\mathrm{Li}}$, существуют многочисленные дефекты в виде мелких ловушек электронов $[1,2,4]$. В кристаллах $\mathrm{LiNbO}_{3}$, выращенных в атмосфере воздуха, всегда присутствуют протоны и атомы водорода в количестве $10^{16}-10^{18} \mathrm{~cm}^{-3}$, связанные с атомами кислорода октаэдров $\mathrm{O}_{6}$ водородными связями. Наличие атомов водорода в структуре приводит к образованию комплексных дефектов типа $V_{\mathrm{Li}} \mathrm{OH}, \mathrm{Nb}_{\mathrm{Li}} \mathrm{OH}$ [4-6]. Присутствие $\mathrm{OH}-$ групп играет важную роль в формировании вторичной структуры и физических характеристик кристалла: повышает низкотемпературную проводимость, понижает эффект фоторефракции и величину коэрцитивного поля [4-6].

Оценить отношение $\mathrm{Li} / \mathrm{Nb}$ в кристалле $\mathrm{LiNbO}_{3}$ и концентрацию имеющихся в нем точечных дефектов $\left(\mathrm{Nb}_{\mathrm{Li}}\right.$ и $\left.V_{\mathrm{Li}}\right)$ можно, определив положение края фундаментального оптического поглощения [7]. Концентрация ОН-групп может быть определена из ИК спектра поглощения в области частот валентных колебаний ОН-групп по методу Клавира [8]. Локализация атомов водорода в структуре кристалла возможна в нескольких позициях. Соответственно в ИК спектре поглощения кристалла будут проявляться несколько линий в области частот валентных колебаний ОН-групп. При уменьшении числа 
возможных позиций атомов водорода в структуре кристалла соответственно уменьшается и число линий в спектре ИК поглощения, соответствующих валентным колебаниям ОН-групп. Этот факт позволяет определить стехиометрию (отношение $\mathrm{Li} / \mathrm{Nb}$ ) кристалла $\mathrm{LiNbO}_{3}$, в том числе и для окрашенных кристаллов, имеющих полосы поглощения в оптическом спектре в области края фундаментального поглощения, вследствие чего для таких кристаллов определение стехиометрии по положению края фундаментального оптического поглощения затруднено.

В настоящей работе, используя подход, изложенный выше, по ИК спектрам поглощения в области частот валентных колебаний водородных связей исследовано влияние состава кристалла на отношение $\mathrm{Li} / \mathrm{Nb}$, концентрацию точечных дефектов $\mathrm{Nb}_{\mathrm{Li}}$ и $V_{\mathrm{Li}}$, концентрацию $\mathrm{OH}^{-}$групп и характер комплексных дефектов, связанных с OH-группами в кристаллах $\mathrm{LiNbO}_{3}$, легированных цинком и магнием $\left(\mathrm{LiNbO}_{3}: \mathrm{Mg}(3.02,5.29,5.91 \mathrm{~mol} \% \mathrm{MgO})\right.$, $\mathrm{LiNbO}_{3}: \mathrm{Zn}(4.54,4.68,6.50 \mathrm{~mol} \% \mathrm{ZnO})$. Результаты исследований сравнивались с результатами, полученными для номинально чистых кристаллов $\mathrm{LiNbO}_{3}$ стехиометрического $\left(\mathrm{LiNbO}_{3 \text { stoich }}\right)$ и конгруэнтного ( $\left.\mathrm{LiNbO}_{3 \text { cong }}\right)$ составов. Кристаллы, легированные цинком и магнием при концентрациях, близких к второму пороговому значению (7.0 и $5.5 \mathrm{~mol} \% \mathrm{ZnO}$ и $\mathrm{MgO}$ соответственно) с низкими эффектом фоторефракции и коэрцитивным полем, перспективны как нелинейно-оптические материалы для преобразования лазерного излучения, в том числе на периодически поляризованных доменах [2-4].

\section{Методика эксперимента}

\section{Выращивание}

$\mathrm{LiNbO}_{3}: \mathrm{Mg}(3.02$,

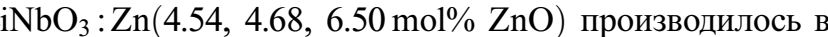
воздушной атмосфере по единой технологии из конгруэнтного расплава методом Чохральского на модернизированной установке „Кристалл-2“, оснащенной системой автоматического контроля диаметра кристалла [9]. Использовалась гранулированная шихта конгруэнтного состава $\left(48.6 \mathrm{~mol} \% \mathrm{Li}_{2} \mathrm{O}\right)$ с высокой насыпной плотностью, полученная методом синтеза-грануляции [10]. Оксид магния (цинка) тщательно перемешивали с шихтой ниобата лития и загружали непосредственно в тигель. С целью снятия термоупругих напряжений выращенные кристаллы подвергались термической обработке при $1200^{\circ} \mathrm{C}$ в течение $24 \mathrm{~h}$. Концентрацию магния (цинка) в кристалле определяли методом атомно-эмиссионной спектрометрии (спектрометр ICPS-9000). Кристаллы $\mathrm{LiNbO}_{3 \text { stoich }}$ выращивались из расплава с $\sim 58.6 \mathrm{~mol} \%$ $\mathrm{Li}_{2} \mathrm{O}$. Более подробно выращивание номинально чистых и сильно легированных кристаллов описано в работе [1]. Монодоменизация кристаллов осуществлялась методом высокотемпературного электродиффузионного отжига при охлаждении образцов со скоростью 20 degrees $/ \mathrm{h}$ в температурном интервале от $\sim 1240-890^{\circ} \mathrm{C}$ в условиях приложения постоянного электрического напряжения. Контроль степени монодоменности осуществлялся методом анализа частотной зависимости электрического импеданса и путем определения статического пьезомодуля $\left(d_{333 \mathrm{c \tau}}\right)$ кристаллической були. Образцы для исследования вырезались из монодоменизированных кристаллов в форме прямоугольных параллелепипедов, ребра которых совпадали по направлению с кристаллофизическими осями $X, Y, Z(Z-$ полярная ось кристалла). Грани параллелепипедов тщательно полировались. Регистрация спектров ИК поглощения производилась в вакууме с помощью спектрометра IFS 66 v/s фирмы Bruker. Обработка ИК спектров и расчеты параметров полос проводились с помощью программ Bomem Grammes V. 2.03, LabSpec 5.5, Origin 8.1.

\section{Результаты и их обсуждение}

На рисунке приведены спектры ИК поглощения исследованных монокристаллов $\mathrm{LiNbO}_{3}$ в области $3420-3550 \mathrm{~cm}^{-1}$, в которой проявляются валентные колебания ОН-групп. В табл. 1 приведены значения параметров спектральных линий. Видно, что спектры всех кристаллов заметно различаются. В ИК спектре поглощения кристалла $\mathrm{LiNbO}_{3 \text { stoich }}$ наблюдаются три полосы с частотами 3465,3480 и $3488 \mathrm{~cm}^{-1}$ (рисунок). Причем ширина полос в спектре кристалла $\mathrm{LiNbO}_{3 \text { stoich }}$ существенно меньше, чем в спектрах других кристаллов (табл. 1). Считается, что полоса с частотой 3465-3466 $\mathrm{cm}^{-1}$ является характерной для стехиометрического кристалла высокой степени структурного совершенства, в структуре которого существует только одна позиция атома водорода $[11,12]$.

В работах $[11,12]$ показано, что с приближением структуры кристалла $\mathrm{LiNbO}_{3}$ к высокосовершенной структуре стехиометрического состава, для которой $\mathrm{Li} / \mathrm{Nb}=1$ и отсутствуют точечные дефекты в виде рядом стоящих одноименных катионов, происходит уменьшение ширины линии с частотой $3466 \mathrm{~cm}^{-1}$ при одновременном уменьшении интенсивностей и ширин полос с частотами 3480 и $3488 \mathrm{~cm}^{-1}$. В ИК спектре поглощения идеального стехиометрического кристалла вообще не должно наблюдаться полос поглощения, связанных с ОН-группами [13]. В ИК спектре реального высокоупорядоченного стехиометрического кристалла, полученного по технологии HTTSSG (high temperature top speed solution growth) из расплава конгруэнтного состава с использованием флюса $\mathrm{K}_{2} \mathrm{O}$, наблюдается только одна узкая полоса $\left(S=3 \mathrm{~cm}^{-1}\right)$ с частотой $3466 \mathrm{~cm}^{-1}[11,12]$.

В работах $[14,15]$ показано, что отношенит интенсивностей линий с частотами 3480 и $3465 \mathrm{~cm}^{-1}$ $\left(I_{\text {rel }}=I_{3480} / I_{3465}\right)$ практически линейно уменьшается с ростом отношения $R=\mathrm{Li} / \mathrm{Nb}$. При $I_{\text {rel }}=0$ интенсивность линии с частотой $3480 \mathrm{~cm}^{-1}$ равна нулю, т.е. эта линия в спектре отсутствует. Исследование интен- 
Таблица 1. Значения частот $\left(v, \mathrm{~cm}^{-1}\right)$, ширин $\left(S, \mathrm{~cm}^{-1}\right)$ и интенсивностей $(I$, rel. units) линий в ИК спектрах поглощения в кристаллах $\mathrm{LiNbO}_{3}$ разного состава при $t=25^{\circ} \mathrm{C}$

\begin{tabular}{|c|c|c|c|c|c|c|c|c|c|c|c|}
\hline \multicolumn{3}{|c|}{$\mathrm{LiNbO}_{3 \text { stoich }}$} & \multicolumn{3}{|c|}{$\mathrm{LiNbO}_{3 \text { cong }}$} & \multicolumn{3}{|c|}{$\mathrm{LiNbO}_{3}: \mathrm{Mg}(3.02)$} & \multicolumn{3}{|c|}{$\mathrm{LiNbO}_{3}: \mathrm{Zn}(4.54)$} \\
\hline$v$ & $I$ & $S$ & $v$ & $I$ & $S$ & $v$ & $I$ & $S$ & $v$ & $I$ & $S$ \\
\hline 3465 & 0.25 & 6.44 & 3467 & 0.04 & 10.22 & 3467 & 0.13 & 12.85 & 3469 & 0.19 & 17.40 \\
\hline 3480 & 0.17 & 7.77 & 3483 & 0.18 & 23.08 & 3481 & 0.28 & 19.02 & 3483 & 0.30 & 16.66 \\
\hline 3488 & 0.06 & 10.19 & 3486 & 0.34 & 27.85 & 3490 & 0.18 & 18.16 & 3492 & 0.18 & 16.48 \\
\hline 3498 & 0.01 & 9.37 & 3490 & 0.06 & 12.51 & 3499 & 0.11 & 16.35 & & & \\
\hline \multicolumn{3}{|c|}{$\mathrm{LiNbO}_{3}: \mathrm{Zn}(4.68)$} & \multicolumn{3}{|c|}{$\mathrm{LiNbO}_{3}: \mathrm{Mg}(5.29)$} & \multicolumn{3}{|c|}{$\mathrm{LiNbO}_{3}: \mathrm{Mg}(5.91)$} & \multicolumn{3}{|c|}{$\mathrm{LiNbO}_{3}: \mathrm{Zn}(6.50)$} \\
\hline$v$ & $I$ & $S$ & $v$ & $I$ & $S$ & $v$ & $I$ & $S$ & $v$ & $I$ & $S$ \\
\hline 3469 & 0.07 & 17.35 & 3526 & 0.15 & 11.47 & 3467 & 0.19 & 13.50 & 3469 & 0.07 & 16.87 \\
\hline 3483 & 0.12 & 14.97 & 3533 & 0.19 & 9.763 & 3483 & 0.37 & 17.37 & 3483 & 0.21 & 17.33 \\
\hline 3489 & 0.08 & 16.95 & 3541 & 0.04 & 11.71 & 3485 & 0.24 & 15.70 & 3491 & 0.07 & 12.99 \\
\hline 3500 & 0.06 & 15.45 & & & & 3499 & 0.44 & 16.14 & 3502 & 0.02 & 12.33 \\
\hline 3527 & 0.08 & 9.03 & & & & & & & 3520 & 0.04 & 12.73 \\
\hline
\end{tabular}

сивностей линий позволяет с меньшей погрешностью $\left(0.01 \mathrm{~mol} \% \mathrm{Li}_{2} \mathrm{O}\right)$, по сравнению с другими методами, определить величину' $R[14,15]$. В то же время, например, определение концентрации $\mathrm{Li}_{2} \mathrm{O}$ по температуре Кюри дает погрешность не менее $0.1 \mathrm{~mol} \% \mathrm{Li}_{2} \mathrm{O}$. Для кристалла $\mathrm{LiNbO}_{3 \text { stoich, }}$ исследованного в данной работе, величина $I_{\text {rel }}=I_{3480} / I_{3465}=0.78$, что дает значение $R=\mathrm{Li} / \mathrm{Nb}=0.98$. Это отличается от данных, полученных в работах [16,17] методом спектроскопии комбинационного рассеяния света (КРС), согласно которым при $R=1$ в спектре КРС кристалла $\mathrm{LiNbO}_{3 \text { stoich }}$ отсутствует малоинтенсивная линия с частотой $120 \mathrm{~cm}^{-1}$, соответствующая двухчастичным состояниям акустических фононов $A_{1}(\mathrm{TO})$-типа симметрии с суммарным волновым вектором, равным нулю. Отличия могут быть обусловлены тем, что линия в спектре КРС с частотой $120 \mathrm{~cm}^{-1}$ чувствительна непосредственно только к изменению отношения $R=\mathrm{Li} / \mathrm{Nb}$ (что автоматически учитывает все дефекты, точечные и комплексные), а полоса с частотой $3466 \mathrm{~cm}^{-1}$ в спектре ИК поглощения чувствительна к концентрации только комплексных дефектов $\mathrm{OH}_{\mathrm{Li}}$. По этой причине значение $R$, определенное по ИК спектрам поглощения, будет меньше значения $R$, определенного по спектрам КРС. Отметим, что метод определения $R$ по спектру ИК поглощения неприменим к легированным кристаллам (например, к кристаллам $\mathrm{LiNbO}_{3}: \mathrm{Mg}(5.29 \mathrm{~mol} \% \mathrm{MgO}$ и др. $\left.)\right)$, в спектре которых нет полос поглощения в области 3465-3466 и $3480 \mathrm{~cm}^{-1}$ (рисунок, кривая 6).

Отношение $\mathrm{Li} / \mathrm{Nb}$ с малой погрешностью (до $0.01 \mathrm{~mol} \%$ ) можно рассчитать также из значения края фундаментального поглощения [7], однако для этого эксперимент необходимо проводить на тонких пластинах (0.5-1 mm) кристаллов $\mathrm{LiNbO}_{3}$. Кроме того, исследуемые кристаллы не должны иметь полос поглощения в области самого края фундаментального поглощения, как это имеет место, например, в кристаллах, легированных катионами $\mathrm{Fe}, \mathrm{Cu}$ и др. В работе [7], используя данные о крае фундаментального оптического поглощения, были предложены эмпирические формулы для оценки отношения $\mathrm{Li} / \mathrm{Nb}$ в номинально чистых кристаллах $\mathrm{LiNbO}_{3}$ (1) или кристаллах, легированных магнием (2),

$$
\begin{gathered}
\frac{\mathrm{Li}}{\mathrm{Nb}}=1-\left(\frac{\lambda_{20}-301.5}{81.29}\right)^{2}, \\
\frac{\mathrm{Li}}{\mathrm{Nb}}=4.887-0.013 \lambda_{20},
\end{gathered}
$$

где $\lambda_{20}$ - край фундаментального оптического поглощения, соответствующий длине волны при коэффициенте поглощения $\alpha=20 \mathrm{~cm}^{-1}$.

Зная величину $\mathrm{Li} / \mathrm{Nb}$, можно рассчитать концентрацию точечных дефектов в кристалле $\mathrm{LiNbO}_{3}$. Согласно модели компенсации Li-вакансий [2], концентрацию точечных дефектов $\mathrm{Nb}_{\mathrm{Li}}^{4+}$ и $V_{\mathrm{Li}}^{-}(\mathrm{mol} \%)$ в кристаллической решетке номинально чистых кристаллов $\mathrm{LiNbO}_{3}$ можно рассчитать [7] по формулам

$$
\begin{gathered}
C\left(V_{\mathrm{Li}}\right)=\left(\frac{4-4 \mathrm{Li} / \mathrm{Nb}}{5+\mathrm{Li} / \mathrm{Nb}}\right) 100, \\
C\left(N b_{\mathrm{Li}}\right)=\frac{C\left(V_{\mathrm{Li}}\right)}{4} .
\end{gathered}
$$

Для кристаллов, легированных магнием, при концентрациях, превышающих пороговую (5.5 и $1.8 \mathrm{~mol} \% \mathrm{MgO}$ для конгруэнтного и стехиометрического кристалла соответственно) справедливы формулы

$$
\begin{gathered}
C\left(V_{\mathrm{Li}}\right)=1 / 2(1-\mathrm{Li} / \mathrm{Nb}) 100, \\
C\left(\mathrm{Nb}_{\mathrm{Li}}\right)=0 .
\end{gathered}
$$

Авторы [7] исходят из предположения, что в структуре сильно легированных кристаллов нет точечных 


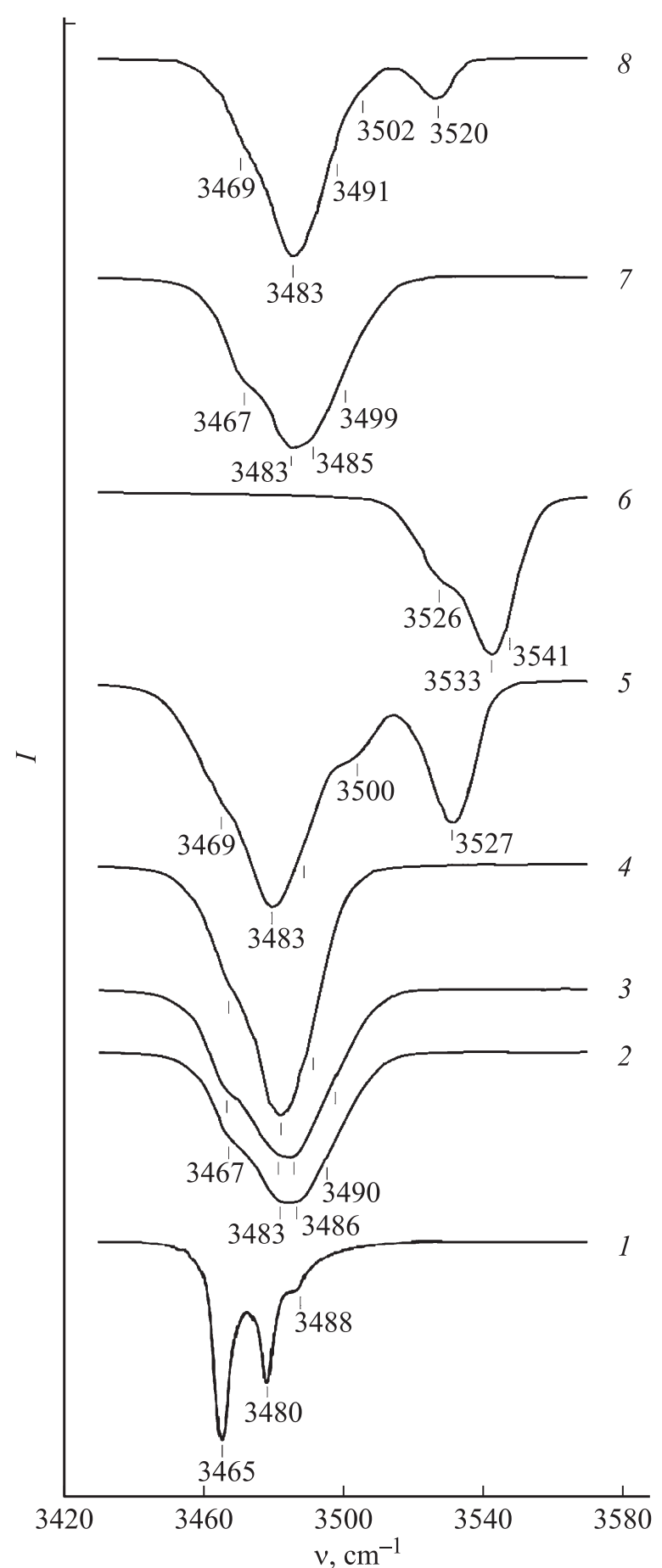

Спектры ИК поглощения кристаллов $\mathrm{LiNbO}_{3}$ в области валентных колебаний ОН-групп: $1-\mathrm{LiNbO}_{3 \text { stoich }}, 2$ $\mathrm{LiNbO}_{3 \text { cong }}, 3-\mathrm{LiNbO}_{3}: \mathrm{Mg} \quad(3.02 \mathrm{~mol} \% \mathrm{MgO}), 4-$ $\mathrm{LiNbO}_{3}: \mathrm{Zn}(4.54 \mathrm{~mol} \% \mathrm{ZnO}), 5-\mathrm{LiNbO}_{3}: \mathrm{Zn}$ (4.68), $6-$ $\mathrm{LiNbO}_{3}: \mathrm{Mg}$ (5.29), $7-\mathrm{LiNbO}_{3}: \mathrm{Mg}$ (5.91), $8-\mathrm{LiNbO}_{3}: \mathrm{Zn}$ (6.50).

дефектов $\mathrm{Nb}_{\mathrm{Li}}$, однако, согласно данным рентгеноструктурного анализа [18], в структуре сильно легированного кристалла $\mathrm{LiNbO}_{3}: \mathrm{Zn}$ при концентрации $4.54 \mathrm{~mol} \% \mathrm{ZnO}$ нет точечных дефектов $\mathrm{Nb}_{\mathrm{Li}}$, но при даль- нейшем увеличении концентрации цинка (кристаллы $\mathrm{LiNbO}_{3}: \mathrm{Zn}$ 4.76-5.19 mol\% $\mathrm{ZnO}$ ) дефекты $\mathrm{Nb}_{\mathrm{Li}}$ вновь появляются [18].

Для определения по ИК спектрам поглощения отношения $\mathrm{Li} / \mathrm{Nb}$ в кристаллах, имеющих полосы поглощения непосредственно в области края фундаментального поглощения, в работе [7] была предложена следующая методика. Для спектра каждого кристалла были вычислены отношения интегральной интенсивности полосы с максимумом при $3466 \mathrm{~cm}^{-1}\left(A_{\text {int,II }}\right)$ к общей интегральной интенсивности ИК спектра поглощения $\left(A_{\text {int,I }}\right): A_{\text {int,II }} / A_{\text {int,I }}=\int \alpha_{1}(v) d v / \int \alpha(v) d v$, где $\alpha(v)$ - коэффициент поглощения в зависимости от длины волны $\left(\mathrm{cm}^{-1}\right)$. На основании построенной зависимости отношений $A_{\text {int,II }} / A_{\text {int,I }}$ от ранее полученных по краю фундаментального поглощения значений $\mathrm{Li} / \mathrm{Nb}$ можно определить отношения $\mathrm{Li} / \mathrm{Nb}$ для случаев, когда кристаллы легированы фотохромными или фоторефрактивными примесями, поглощающими в видимой и УФ областях спектра [7]. Очевидно, эту методику определения отношения $\mathrm{Li} / \mathrm{Nb}$ можно применить и для других кристаллов $\mathrm{LiNbO}_{3}$, например для образцов толщиной $>1 \mathrm{~mm}$, для которых невозможно измерить край фундаментального оптического поглощения $\lambda_{20}$. В настоящей работе исследованные образцы имели разную толщину $(Z=4-10 \mathrm{~mm})$. Результаты наших расчетов отношения $\mathrm{Li} / \mathrm{Nb}$ (определенного по ИК спектру поглощения по методике [7]) и концентрации точечных дефектов $\mathrm{Nb}_{\mathrm{Li}}^{4+}$ и $V_{\mathrm{Li}}^{-}$в исследованных кристаллах $\mathrm{LiNbO}_{3}$ представлены в табл. 2.

Из рисунка видно, что ИК спектры кристаллов

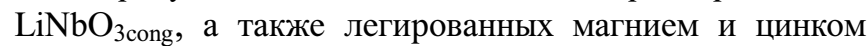
кристаллов $\mathrm{LiNbO}_{3}$ значительно отличаются от ИК спек-

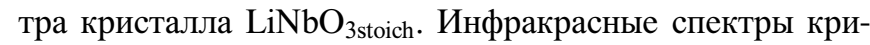
сталла $\mathrm{LiNbO}_{3 \text { cong }}$ и кристаллов $\mathrm{LiNbO}_{3}: \mathrm{Mg}(3.02 \mathrm{~mol} \%$ $\mathrm{MgO}), \quad \mathrm{LiNbO}_{3}: \mathrm{Zn}(4.54 \mathrm{~mol} \% \mathrm{ZnO})$ в области первой пороговой концентрации легирующего элемента ( 4.0 mol\% $\mathrm{ZnO}$ и $3 \mathrm{~mol} \% \mathrm{MgO}[1,2,19])$ представляют собой размытую полосу поглощения, состоящую из нескольких компонентов одинаковой поляризации с частотами $3467\left(v_{1}\right), 3483\left(v_{2}\right), 3486\left(v_{3}\right) \mathrm{cm}^{-1}$ и слабой полосы с частотой $\sim 3490 \mathrm{~cm}^{-1}\left(v_{4}\right)$. Полосу с частотой $3480-3485 \mathrm{~cm}^{-1}$ считают характерной для конгруэнтного кристалла и относят к валентным колебаниям комплекса $V_{\mathrm{Li}} \mathrm{OH}$ [20]. Так как в идеальной структуре кристалла $\mathrm{LiNbO}_{3}$ стехиометрического состава нет дефектов $\mathrm{Nb}_{\mathrm{Li}}$, то нет и дефектов $V_{\mathrm{Li}}$. Это значит, что в ИК спектре не должно быть полосы с частотой $3480-3485 \mathrm{~cm}^{-1}$, что действительно подтверждается экспериментально $[11,12]$.

В структуре конгруэнтного кристалла присутствует $\sim 1 \mathrm{~mol} \%$ дефектов $\mathrm{Nb}_{\mathrm{Li}}$ и $\sim 4 \mathrm{~mol} \%$ дефектов $V_{\mathrm{Li}}[2]$. При легировании конгруэнтного кристалла катионами $\mathrm{Zn}$ и $\mathrm{Mg}$ будет происходить вытеснение из структуры дефектов $\mathrm{Nb}_{\mathrm{Li}}$, при этом вследствие сохранения зарядовой нейтральности катионной подрешетки в целом будет изменяться количество дефектов $V_{\mathrm{Li}}[2,4]$. Эти изменения 
структуры можно проследить по ИК спектрам поглощения. В структуре кристалла $\mathrm{LiNbO}_{3}: \mathrm{Mg}(3.02 \mathrm{~mol} \%$ $\mathrm{MgO})$ присутствуют дефекты $\mathrm{Nb}_{\mathrm{Li}}$ и $V_{\mathrm{Li}}[2]$, и в ИК спектре, как и для кристалла $\mathrm{LiNbO}_{3 \text { cong, }}$ в области $3480-3486 \mathrm{~cm}^{-1}$ наблюдается две полосы поглощения (рисунок). В спектре кристалла $\mathrm{LiNbO}_{3}: \mathrm{Zn}(4.54 \mathrm{~mol} \%$ $\mathrm{ZnO})$ в области $3480-3486 \mathrm{~cm}^{-1}$ уверенно наблюдается только одна асимметричная полоса поглощения (рисунок, кривая 4). По результатам полнопрофильного рентгеноструктурного анализа в структуре кристалла $\mathrm{LiNbO}_{3}: \mathrm{Zn}(4.54 \mathrm{~mol} \% \mathrm{ZnO})$ нет дефектов $\mathrm{Nb}_{\mathrm{Li}}$, но при дальнейшем увеличении концентрации $\mathrm{Zn}$ дефекты $\mathrm{Nb}_{\mathrm{Li}}$ вновь появляются [18], и в ИК спектре присутствует уже несколько полос поглощения (рисунок, кривая 5).

Из рисунка видно, что ИК спектры поглощения кристаллов $\mathrm{LiNbO}_{3}: \mathrm{Mg}$ и $\mathrm{LiNbO}_{3}: \mathrm{Zn}$ значительно различаются по количеству линий, их интенсивностям и значениям частот. Видно, что спектр кристалла $\mathrm{LiNbO}_{3}: \mathrm{Mg}(5.29 \mathrm{~mol} \% \mathrm{MgO})$ в целом существенно ( на $50 \mathrm{~cm}^{-1}$ ) сдвинут в высокочастотную область и представлен тремя сильно перекрывающимися полосами с частотами 3526,3535 и $3541 \mathrm{~cm}^{-1}$ (рисунок). Однако при дальнейшем увеличении содержания магния до $5.91 \mathrm{~mol} \%$ ИК спектр в целом смещается обратно в низкочастотную область и приобретает вид, характер-

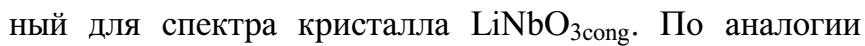
с кристаллом $\mathrm{LiNbO}_{3}: \mathrm{Zn}$ можно предположить, что в структуре кристалла $\mathrm{LiNbO}_{3}: \mathrm{Mg}(5.29 \mathrm{~mol} \% \mathrm{MgO})$ нет дефектов $\mathrm{Nb}_{\mathrm{Li}}$ и $V_{\mathrm{Li}}$, а в структуре кристалла $\mathrm{LiNbO}_{3}: \operatorname{Mg}(5.91 \mathrm{~mol} \% \mathrm{MgO})$ дефекты $\mathrm{Nb}_{\mathrm{Li}}$ и $V_{\mathrm{Li}}$ снова появляются.

В то же время изменения ИК спектра при увеличении содержания цинка носят совершенно другой характер (рисунок, кривые 5,8). В области $3465-3489 \mathrm{~cm}^{-1}$ в спектре не происходит исчезновение полос, характерное для кристаллов, легированных магнием, но при этом в высокочастотной области появляются дополнительные полосы поглощения с частотой $3520-3527 \mathrm{~cm}^{-1}$ (рисунок, кривые 5,8). Такие различия в спектрах кристаллов $\mathrm{LiNbO}_{3}: \mathrm{Mg}$ и $\mathrm{LiNbO}_{3}: \mathrm{Zn}$ очевидно обусловлены разным характером вхождения примесей $\mathrm{Mg}$ и $\mathrm{Zn}$ в структуру кристалла ниобата лития. Вхождение $\mathrm{Mg}$ имеет „поэтапный“ (скачкообразный) характер сначала магний полностью вытесняет дефекты $\mathrm{Nb}_{\mathrm{Li}}$ и только затем он начинает замещение атомов $\mathrm{Li}$ и $\mathrm{Nb}$. При вхождении цинка в структуру кристалла вытеснение им дефектов $\mathrm{Nb}_{\mathrm{Li}}$ и замещение атомов $\mathrm{Li}$ и $\mathrm{Nb}$ идут одновременно. Причем процесс замещения носит плавный характер [2]. Необходимо отметить, что полосы поглощения в высокочастотной области $\left(3520-3535 \mathrm{~cm}^{-1}\right)$ относятся к колебаниям комплексов $\mathrm{Me}_{\mathrm{Nb}}-\mathrm{OH}_{\mathrm{Li}}(\mathrm{Me}-$ легирующий катион), причем в работе [11] показано, что значение смещения ИК спектра в высокочастотную область зависит от валентности легирующего металла: чем меньше валентность, тем слабее водородная связь, тем больше частота соответствующего ОН-колебания.
Из спектра ИК поглощения можно рассчитать концентрацию ОН-групп по методу Клавира [8] по формуле

$$
C(\mathrm{OH})=\frac{A_{\text {int,I }}}{(\ln 10) \alpha_{\mathrm{OH}}},
$$

где $\mathrm{C}(\mathrm{OH})$ - концентрация дефектов $\mathrm{OH}$ в образце $\left(\mathrm{cm}^{-3}\right), A_{\text {int,I }}-$ общая интегральная интенсивность ИК спектра в области валентных колебаний ОН-групп: $\left.A_{\text {int,I }}=\int \alpha(v) d v\right)$, где $\alpha(v)-$ коэффициент поглощения в зависимости от длины волны, $\mathrm{cm}^{-1}, a_{\mathrm{OH}}-$ интенсивность поглощения иона, равная $(9.125 \pm 1.369) \cdot 10^{-18} \mathrm{~cm}[8,21]$.

Результаты расчета представлены в табл. 2. Так как исследованные кристаллы имели разную толщину $(Z=4-10 \mathrm{~mm})$, то результаты расчета концентрации ОН-групп были нормированы на толщину образцов. Из табл. 2 видно, что в легированных кристаллах при концентрации легирующего элемента вблизи второго концентрационного порога (кристаллы $\mathrm{LiNbO}_{3}: \mathrm{Mg}(5.29 \mathrm{~mol} \% \mathrm{MgO})$ и $\mathrm{LiNbO}_{3}: \mathrm{Zn}(4.68$ и $6.50 \mathrm{~mol} \% \mathrm{ZnO})$ ) количество ОН-групп минимальное. Оно меньше, чем количество ОН-групп в кристалле

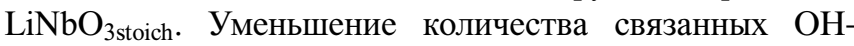
групп, вероятно, приводит к увеличению количества свободных протонов в сильно легированных кристаллах $\mathrm{LiNbO}_{3}: \mathrm{Mg}$ и $\mathrm{LiNbO}_{3}: \mathrm{Zn}$, что может обусловливать более высокие электропроводность и скорость термической фиксации голограмм в этих кристаллах по сравнению с кристаллом $\mathrm{LiNbO}_{3 \text { cong }}[2,4]$, а также снижение эффекта фоторефракции.

В работе [22] методом компьютерного моделирования был проведен анализ возможных позиций атомов водорода в стехиометрических кристаллах ниобата лития. Показано, что все гипотетические позиции атомов водорода, расположенные в середине О-О-связей (как в идеальной бездефектной структуре, так и в структуре, в которой присутствуют дефекты $\left.V_{\mathrm{Li}}\right)$ в ближайшей окрестности дефекта $V_{\mathrm{Li}}$ являются неустойчивыми и должны быть исключены из дальнейшего рассмотрения. При этом позиция атома водорода на оси $c$ не подтвердилась. Анализ возможных траекторий перемещения атомов водорода в структуре кристалла $\mathrm{LiNbO}_{3}$ показывает, что атом водорода в стехиометрической структуре ассоциирован только с одним из ионов кислорода в верхнем кислородном треугольнике октаэдра $\mathrm{NbO}_{6}$. При этом ориентация дипольного момента ОН-группы несколько отличается от направления короткой $\mathrm{O}-\mathrm{O}-$ связи. Таким образом, образование водородной связи приводит не только к кардинальному изменению волновых функций внешних электронных орбиталей иона кислорода и параметров его электронной поляризуемости, но и к сильному искажению всего октаэдра $\mathrm{NbO}_{6}$ [22]. Такие изменения структуры кристаллов можно исследовать по спектрам КРС в области 850-900 $\mathrm{cm}^{-1}$, отвечающей валентным мостиковым колебаниям атомов кислорода $\mathrm{B}-\mathrm{O}-\mathrm{B}(\mathrm{B}-\mathrm{Nb}$ или легирующий металл) в октаэдре 
Таблица 2. Отношение $\mathrm{Li} / \mathrm{Nb}$ (определенное по ИК спектру поглощения), общая интегральная интенсивность ИК спектра в области валентных колебаний ОН-групп $\left(A_{\text {int,I }}\right)$, интегральная интенсивность полосы с частотой $3466-3469 \mathrm{~cm}^{-1}\left(A_{\text {int,II }}\right)$, толщина $(d)$ кристаллов вдоль оси $Z$, концентрация ОН-групп $\left(C_{\mathrm{OH}}^{-}\right)$и точечных дефектов $\mathrm{Nb}_{\mathrm{Li}}^{4+}$ и $V_{\mathrm{Li}}^{-}$в кристаллах ниобата лития разного состава при $t=25^{\circ} \mathrm{C}$

\begin{tabular}{c|c|c|c|c|c|c|c|c}
\hline Кристалл & $d, \mathrm{~mm}$ & $\mathrm{Li} / \mathrm{Nb}$ & $C\left(\mathrm{Nb}_{\mathrm{Li}}\right), \mathrm{mol} \%$ & $C\left(V_{\mathrm{Li}}^{-}\right), \mathrm{mol} \%$ & $A_{\text {int,I }}$ & $A_{\text {int,II }}$ & $A_{\text {int,II }} / A_{\text {int,I }}$ & $C_{\mathrm{OH}}^{-}, \mathrm{cm}^{-3}$ \\
\hline $\mathrm{LiNbO}_{\text {3cong }}$ & 4 & 0.946 & 0.91 & 3.63 & 6.860 & 0.513 & 0.075 & $8.15 \cdot 10^{16}$ \\
$\mathrm{LiNbO}_{3 \text { stoich }}$ & 4 & 1.000 & 0 & 0 & 3.380 & 1.952 & 0.578 & $4.03 \cdot 10^{16}$ \\
$\mathrm{LiNbO}_{3}: \mathrm{Mg}(3.02)$ & 4 & 0.970 & 0.5 & 2.01 & 12.580 & 2.424 & 0.189 & $1.50 \cdot 10^{17}$ \\
$\mathrm{LiNbO}_{3}: \mathrm{Zn}(4.54)$ & 10 & 0.950 & 0 & 2.50 & 12.829 & 1.346 & 0.105 & $6.11 \cdot 10^{16}$ \\
$\mathrm{LiNbO}_{3}: \mathrm{Zn}(4.68)$ & 10 & 0.975 & 0 & 1.25 & 7.018 & 1.450 & 0.207 & $3.34 \cdot 10^{16}$ \\
$\mathrm{LiNbO}_{3}: \mathrm{Mg}(5.29)$ & 10 & - & 0 & - & 5.826 & - & - & $2.77 \cdot 10^{16}$ \\
$\mathrm{LiNbO}_{3}: \mathrm{Mg}(5.91)$ & 10 & 0.972 & 0 & 1.40 & 16.459 & 3.170 & 0.193 & $7.83 \cdot 10^{16}$ \\
$\mathrm{LiNbO}_{3}: \mathrm{Zn}(6.50)$ & 10 & 0.970 & 0 & 1.50 & 7.137 & 1.346 & 0.189 & $4.00 \cdot 10^{16}$
\end{tabular}

$\mathrm{NbO}_{6}$ вдоль полярной оси [2]. В работе [2] при исследовании спектров КРС кристаллов $\mathrm{LiNbO}_{3}$ разного состава было показано, что в спектрах номинально чистых и легированных небольшим количеством примесей (в частности, $0.062-3.5 \mathrm{~mol} \% \mathrm{MgO}$ и $0.23 \mathrm{~mol} \% \mathrm{ZnO}$ ) в области $850-900 \mathrm{~cm}^{-1}$ наблюдается только одна линия, ширина которой зависит от величины $\mathrm{Li} / \mathrm{Nb}$. При увеличении содержания в структуре кристаллов легирующего элемента $(3.5-4.5 \mathrm{~mol} \% \mathrm{MgO}[2]$ и $2.27-5.84 \mathrm{~mol} \% \mathrm{ZnO}[2,18])$ в области 850-900 $\mathrm{cm}^{-1}$, наблюдаются две линии с частотами 873 и $\sim 900 \mathrm{~cm}^{-1}$. То есть характер мостиковой связи В-О-В определяется не только жесткостью ее мостиков В-O, но и положением катионов В (B $\mathrm{Nb}$ или легирующий металл) в октаэдрах. Двухмодовое поведение линии в области $850-900 \mathrm{~cm}^{-1}$ обусловлено тем, что катионы $\mathrm{Nb}$ или легирующего металла в разных октаэдрах расположены в разных позициях, концентрация их достаточно велика, и они слабо взаимодействуют между собой [2]. Изменения в спектрах КРС в области валентных мостиковых колебаний сильно легированных кристаллов $\mathrm{LiNbO}_{3}$ коррелируют с изменениями, наблюдающимися в ИК спектрах (рисунок, кривые 5, 6,8). Это подтверждает результаты теоретической работы [22] и экспериментальных исследований $[11,12]$, в которых показано, что атомы водорода в структуре кристалла $\mathrm{LiNbO}_{3}$ образуют водородные связи с одним из ионов кислорода октаэдра $\mathrm{NbO}_{6}$, расположенным на полярной оси.

\section{Заключение}

Таким образом, по ИК спектрам поглощения в области валентных колебаний ОН-групп рассчитана концен-

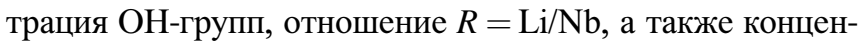
трация точечных дефектов $\mathrm{Nb}_{\mathrm{Li}}^{4+}$ и $V_{\mathrm{Li}}^{-}$в кристаллической решетке номинально чистых кристаллов $\mathrm{LiNbO}_{3}$ стехиометрического и конгруэнтного составов, а также кристаллов, легированных магнием и цинком при концентрациях, близких к пороговым значениям. Показано, что, согласно нашим расчетам, величина $R$ равна еди- нице для стехиометрического кристалла ( $\left.\mathrm{LiNbO}_{3 \text { stoich}}\right)$, выращенного из расплава с $\sim 58.6 \mathrm{~mol}_{0} \mathrm{Li}_{2} \mathrm{O}$, а для конгруэнтного кристалла равна 0.946 , что подтверждается фазовой диаграммой ниобата лития и хорошо совпадает с определением величины $R$ по температуре Кюри. Расчет количества ОН-групп показал минимальное содержание атомов водорода в легированных кристаллах $\mathrm{LiNbO}_{3}: \mathrm{Mg}$ и $\mathrm{LiNbO}_{3}: \mathrm{Zn}$ при концентрации легирующего элемента вблизи второго концентрационного порога $(5.5 \mathrm{~mol} \% \mathrm{MgO}$ и $7.0 \mathrm{~mol} \% \mathrm{ZnO})$. При этом в сильно легированных кристаллах $\mathrm{LiNbO}_{3}: \mathrm{Mg}$ и $\mathrm{LiNbO}_{3}: \mathrm{Zn}$ свободных протонов (вносящих вклад в проводимость) больше, чем в слабо легированных и номинально чистых кристаллах, что может обусловливать более высокие электропроводность, скорость термической фиксации голограмм и снижение эффекта фоторефракции. Наблюдается корреляция в поведении параметров полос в ИК спектрах поглощения сильно легированных кристаллов $\mathrm{LiNbO}_{3}: \mathrm{Mg}$ и $\mathrm{LiNbO}_{3}: \mathrm{Zn}$ и в поведении линии спектра КРС, отвечающей валентным мостиковым колебаниям атомов кислорода в октаэдре $\mathrm{NbO}_{6}$ вдоль полярной оси. Это подтверждает результаты работ [11,12,22,23], в которых показано, что атомы водорода в кристалле $\mathrm{LiNbO}_{3}$ образуют водородные связи с одним из ионов кислорода октаэдра $\mathrm{NbO}_{6}$, расположенным на полярной оси кристалла.

\section{Конфликт интересов}

Авторы заявляют, что у них нет конфликта интересов.

\section{Список литературы}

[1] Палатников М.Н., Сидоров Н.В., Макарова О.В., Бирюкова И.В. Фундаментальные аспекты технологии сильно легированных кристаллов ниобата лития. Апатиты: Издво КНЦ РАН, 2017. $241 \mathrm{c}$.

[2] Сидоров Н.В., Волк Т.Р., Маврин Б.Н., Калинников В.Т. Ниобат лития: дефекты, фоторефракция, колебательный спектр, поляритоны. М.: Наука, 2003. 255 с. 
[3] Shur V.Ya., Akhmatkhanov A.R., Baturin I.S. // Appl. Phys. Rew. 2015. V. 2. N 4. P. 040604. doi org/10.1063/1.4928591

[4] Volk T., Wohlecke M. Lithium Niobate. Defects, Photorefraction and Ferroelectric Switching. Berlin: Springer, 2008. 250 p.

[5] Cabrera J.M, Olivares J., Carrascosa M., Rams J., Müller R., Diéguez E. // Adv. Phys. 1996. V. 45. N 5. P. 349. doi 10.1080/00018739600101517

[6] Kovacs L., Wöhlecke M., Jovanovic A., Polgar K., Kapphan S. // J. Phys. Chem. Sol. 1991. V. 52. N 6. P. 797. doi.org/10.1016/0022-3697(91)90078-E

[7] Саллум М.И., Грунский О.С., Маньшина А.А., Тверьянович А.С., Тверьянович Ю.С. // Изв. РАН. Сер. хим. 2009. T. 73. № 11. C. 2162; Salloum M.Y., Grunsky O.S., Man'shina A.A., Tver'yanovich A.S., Tver'yanovich Yu.S. // Russ. Chem. Bull., Int. Ed. 2009. V. 58. P. 2228.

[8] Klauer S., Wöhlecke M., Kapphan S. // Phys. Rev. B. 1992. V. 45. P. 2786. doi.org/10.1103/PhysRevB.45.2786

[9] Palatnikov M.N., Birukova I.V., Masloboeva S.M., Makarova O.V., Manukovskaya D.V., Sidorov N.V. // J. Cryst. Growth. 2014. V. 386. P. 113. doi $10.1016 /$ j.jcrysgro.2013.09.038

[10] Палатников М.Н., Сидоров Н.В., Бирюкова И.В., Щербина О.Б., Калинников В.Т. // Персп. матер. 2011. № 2. C. 93.

[11] Lengyel K., Peter A., Kovacs L., Corradi G., Palfavi L, Hebling J., Unferdorben M., Dravecz G., Hajdara I., Szaller Zs., Polgar K. // Appl. Phys. Rev. 2015. V. 2. P. 040601. doi.org/10.1063/1.4929917

[12] Polgar K., Peter A., Kovacs L., Corradi G., Szaller Zs. // J. Cryst. Growth. 1997. V. 177. N 3-4. P. 211. doi 10.1016/S0022-0248(96)01098-6

[13] Gröne A., Kapphan S. // J. Phys. Chem. Sol. 1995. V. 56. N 5. P. 687. doi.org/10.1016/0022-3697(94)00184-7A

[14] Dravecz G., Kovács L. // Appl. Phys. B. 2007. V. 88. N 2. P. 305. doi.org/10.1007/s00340-007-2704-9

[15] Dravecz G., Kovács L., Peter A., Polgar K., Bourson P. // Phys. Stat. Sol. C. 2007. V. 4. N 3. P. 1313. doi.org/10.1002/pssc.200673733

[16] Okamoto Y., Wang P.-C., Scott J.F. // Phys. Rev. B. 1985. V. 32. N 10. P. 6787. doi.org/10.1103/PhysRevB.32.6787

[17] Сидоров Н.В., Палатников М.Н., Калинников В.Т. // Опт. и спектр. 1997. Т. 82. № 1. С. 38.

[18] Сидоров Н.В., Теплякова Н.А., Яничев А.А., Палатников М.Н., Макарова О.В., Алешина Л.А., Кадетова A.B. // Неорг. матер. 2017. Т. 53. № 5. С. 491. doi 10.7868/S0002337X17050177; Sidorov N.V., Teplyakova N.A., Yanichev A.A., Palatnikov M.N., Makarova O.V., Aleshina L.A., Kadetova A.V. // Inorg. Mater. 2017. V. 53. 5. P. 489. doi 10.1134/S002016851705017X

[19] Palatnikov M.N., Biryukova I.V., Makarova O.V., Sidorov N.V., Efremov V.V., Efremov I.N., Teplyakova N.A., Manukovskaya D.V. // Advanced Materials - Manufacturing, Physics, Mechanics and Applications / Ed. by Parinov I.A., Shun-Hsyung, Topolov V.Yu. Heidelberg, New York, Dordrecht, London: Springer, 2016. V. 175. P. 87-99. http://www.springer.com/gp/book/9783319263229

[20] Iyi N., Kitamura K., Izumi F., Yamamoto J.K., Hayashi T., Asano H., Kimura S. // J. Sol. Stat. Chem. 1992. V. 101. N 2. P. 340. doi 10.1016/0022-4596(92)90189-3

[21] Watanabe Y., Sota T., Suzukits K., Iyi N., Kimura S. // J. Phys.: Cond. Matter. 1995. V. 7. N 18. P. 3627. doi.org/10.1088/0953-8984/7/18/025
[22] Евдокимов С.В., Яценко А.В. // Кристаллогр. 2003. Т. 48. № 4. C. 594; Yevdokimov S.V., Yatsenko A.V. // Cryst. Rep. 2003. V. 48. N 4. P. 542. doi $10.1134 / 1.1595175$

[23] Kong Y., Xu J., Zhang W., Zhang G. // J. Phys. Chem. Sol. 2000. V. 61. N 8. P. 1331. doi.org/10.1016/S00223697(99)00413-8 